\title{
Hausdorff Dimension and Fractal Dimension of the Global Attractor for the Higher-Order Coupled Kirchhoff-Type Equations
}

\author{
Guoguang Lin, Sanmei Yang \\ Department of Mathematics, Yunnan University, Kunming, China \\ Email: gglin@ynu.edu.cn
}

How to cite this paper: Lin, G.G. and Yang, S.M. (2017) Hausdorff Dimension and Fractal Dimension of the Global Attractor for the Higher-Order Coupled Kirchhoff-Type Equations. Journal of Applied Mathematics and Physics, 5, 2411-2424. https://doi.org/10.4236/jamp.2017.512197

Received: November 28, 2017 Accepted: December 26, 2017 Published: December 29, 2017

Copyright (c) 2017 by authors and Scientific Research Publishing Inc. This work is licensed under the Creative Commons Attribution International License (CC BY 4.0). http://creativecommons.org/licenses/by/4.0/

\begin{abstract}
This paper mainly deals with the higher-order coupled Kirchhoff-type equations with nonlinear strong damped and source terms in a bounded domain. We obtain some results that are estimation of the upper bounds of Hausdorff dimension and Fractal dimension of the global attractor.
\end{abstract}

\section{Keywords}

Higher-Order Coupled Kirchhoff-Type Equations, Source Term, Hausdorff Dimension, Fractal Dimension, Nonlinear Dissipation

\section{Introduction}

Guoguang Lin and Sanmei Yang [1] had studied the existence and uniqueness of the solution and global attractors for the higher-order coupled Kirchhoff-type equations. Furthermore, we consider the Hausdorff dimension and Fractal dimension of the global attractor for the following Hinder-order coupled Kirchhoff equations:

$$
\begin{gathered}
u_{t t}+M\left(\|\nabla u\|^{2}+\|\nabla v\|^{2}\right)(-\Delta)^{m} u+\beta(-\Delta)^{m} u_{t}+g_{1}(u, v)=f_{1}(x), \\
v_{t t}+M\left(\|\nabla u\|^{2}+\|\nabla v\|^{2}\right)(-\Delta)^{m} v+\beta(-\Delta)^{m} v_{t}+g_{2}(u, v)=f_{2}(x), \\
u(x, 0)=u_{0}(x), u_{t}(x, 0)=u_{1}(x), x \in \Omega, \\
v(x, 0)=v_{0}(x), v_{t}(x, 0)=v_{1}(x), x \in \Omega, \\
\left.u\right|_{\partial \Omega}=0, \frac{\partial^{i} u}{\partial \mu^{i}}=0, i=1,2,3, \cdots, m-1,
\end{gathered}
$$




$$
\left.v\right|_{\partial \Omega}=0, \frac{\partial^{i} v}{\partial v^{i}}=0, i=1,2,3, \cdots, m-1,
$$

where $m>1$ is an integer constant and $\Omega$ is a bounded domain of $R^{n}$ with a smooth Dirichlet boundary $\partial \Omega$ and initial value. $\mu_{i}$ and $v_{i}$ are the unit outward normal on $\partial \Omega, M(s)$ is a nonnegative $C^{1}$ function, $(-\Delta)^{m} u_{t}$ and $(-\Delta)^{m} v_{t}$ are strongly damping, $g_{1}(u, v)$ and $g_{2}(u, v)$ are nonlinear source terms, $f_{1}(x)$ and $f_{2}(x)$ are given forcing function.

When considering single Higher-order Kirchhoff-type equation, $\beta=\sigma\left(\left\|\nabla^{m} u\right\|^{2}\right), g(u, v)=0$ and $m=1$ in $M(s)$, becomes following Higher-order Kirchhoff-type wave equation with nonlinear strongly damping:

$$
\begin{gathered}
u_{t t}+\sigma\left(\left\|\nabla^{m} u\right\|^{2}\right)(-\Delta)^{m} u_{t}+\phi\left(\left\|\nabla^{m} u\right\|^{2}\right)(-\Delta)^{m} u=f(x), \\
u(x, t)=0, \frac{\partial^{i} u}{\partial v^{i}}=0, i=1, \cdots, m-1, x \in \partial \Omega, t \in(0,+\infty), \\
u(x, 0)=u_{0}(x), u_{t}(x, 0)=u_{1}(x), x \in \Omega .
\end{gathered}
$$

This equation had been studied some main results that are existence and uniqueness of the solution in $H^{2 m}(\Omega) \times H_{0}^{m}(\Omega)$ and global attractors by Yuting Sun, Yunlong Gao and Guoguang Lin, see [2].

In case of $\beta=1$ and $m=1$ in $M(s)$, the Equation (1.1) becomes a Higher-order Kirchhoff-type equation with nonlinear strongly dissipation and source term:

$$
\begin{gathered}
u_{t t}+(-\Delta)^{m} u_{t}+M\left(\left\|(-\Delta)^{\frac{m}{2}} u\right\|^{2}\right)(-\Delta)^{m} u+g(u)=f(x), x \in \Omega, t>0, m>1 \\
u(x, t)=0, \frac{\partial^{i} u}{\partial v^{i}}=0, i=1, \cdots, m-1, x \in \partial \Omega, x \in \Omega, t>0 \\
u(x, 0)=u_{0}(x), u_{t}(x, 0)=u_{1}(x) .
\end{gathered}
$$

This equation had been investigated the existence and uniqueness of the solution, global attractors and estimation Hausdorff and fractal dimensions of the global attractor by Chen, Wei Wang and Guoguang Lin, see [3]. As for the study of estimation Hausdorff dimension of the global attractor, we applied different method from theirs.

Under the situation of $\beta=1$ and $M(s)=\left(\alpha+\beta\left\|\nabla^{m} u\right\|^{2}\right)^{q}$, the problem (1.1) becomes a class of strongly damped Higher-order Kirchhoff-type equation:

$$
\begin{gathered}
u_{t t}+(-\Delta)^{m} u_{t}+\left(\alpha+\beta\left\|\nabla^{m} u\right\|^{2}\right)^{q}(-\Delta)^{m} u+g(u)=f(x),(x, t) \in \Omega \times[0,+\infty), \\
u(x, 0)=u_{0}(x), u_{t}(x, 0)=u_{1}(x), x \in \Omega, \\
u(x, t)=0, \frac{\partial^{i} u}{\partial v^{i}}=0, i=1, \cdots, m-1, x \in \partial \Omega, t \in(0,+\infty) .
\end{gathered}
$$

This equation had been studied the existence and uniqueness of the solution, global attractors and estimation of the upper bounds of Hausdorff for the global 
attractors and the existence of a fractal exponential attractor with non-supercritical and critical cases by Guoguang Lin and Yunlong Gao, see [4]. Their novelty is that it overcomes $\left(\alpha+\beta\left\|\nabla^{m} u\right\|^{2}\right)^{q}$ by using generalized Gronwall's inequality in Lemma 2 .

Next, the main purpose of this paper is to study a precise estimation of upper bounds of Hausdorff dimension and Fractal dimension of the global attractor.

\section{Preliminaries}

To better carry out our work, We denote the some simple symbol, $\|\cdot\|$ represents norm, (,) stands for inner product and $H^{m}(\Omega)=H^{m}(\Omega)$, $H_{0}^{m}(\Omega)=H^{m}(\Omega) \cap H_{0}^{1}(\Omega), H_{0}^{2 m}(\Omega)=H^{2 m}(\Omega) \cap H_{0}^{1}(\Omega)$, $f_{i}=f_{i}(x)(i=1,2), \quad H(\Omega)=L^{2}(\Omega),\|\cdot\|=\|\cdot\|_{L^{2}}, \quad\|\cdot\|_{\infty}=\|\cdot\|_{L^{\infty}}, \quad v=\|\nabla u\|^{2}+\|\nabla v\|^{2}$. $c_{i}(i=1, \cdots), \mu_{i}(i=0,1)$ are constants. $\lambda_{1}^{m}$ is the first eigenvalue of the operator $(-\Delta)^{m}$.

Next, we give some assumptions needed for problem (1.1)-(1.6).

(H1) $M(s) \in C^{2}(\Omega), M^{\prime}(s) \geq S_{0} \geq 0$.

(H2) $0 \leq \mu_{0} \leq M(v) \leq \mu_{1}, \quad \mu=\left\{\begin{array}{l}\mu_{0}, \frac{\mathrm{d}}{\mathrm{d} t}\left(\left\|\nabla^{m} \theta\right\|^{2}+\left\|\nabla^{m} \sigma\right\|^{2}\right) \geq 0, \\ \mu_{1}, \frac{\mathrm{d}}{\mathrm{d} t}\left(\left\|\nabla^{m} \theta\right\|^{2}+\left\|\nabla^{m} \sigma\right\|^{2}\right)<0,\end{array}\right.$

(H3) $\forall M>0$, there exists $k_{1}, k_{2}$, such that

$$
\begin{gathered}
\left\|g_{\text {iu }}(\tilde{u}, \tilde{v})-g_{\text {iu }}(u, v)\right\|_{L^{\infty}} \leq k_{1}\left(\|\tilde{u}-u\|^{k}+\|\tilde{v}-v\|^{k+1}\right) . \\
\left\|g_{i v}(\tilde{u}, \tilde{v})-g_{i v}(u, v)\right\|_{L^{\infty}} \leq k_{2}\left(\|\tilde{u}-u\|^{k+1}+\|\tilde{v}-v\|^{k}\right) . \\
g_{i}(u, v) \in C^{1}(\Omega) .
\end{gathered}
$$

Lemma 2.1. (Young's inequality [5]) For any $\varepsilon>0$ and $a, b \geq 0$, then

$$
a b \leq \frac{\varepsilon^{p}}{p} a^{p}+\frac{b^{q}}{q \varepsilon^{q}},
$$

where $\frac{1}{p}+\frac{1}{q}=1, p>1, q>1$.

Lemma 2.2. (Gronwall's inequality [5]) If $\forall t \in\left[t_{0},+\infty\right), y(t) \geq 0$ and $\frac{\mathrm{d} y}{\mathrm{~d} t}+g y \leq h$, such that

$$
y(t) \leq y\left(t_{0}\right) \mathrm{e}^{-g\left(t-t_{0}\right)}+\frac{h}{g}, t \geq t_{0},
$$

where $g \geq 0, h \geq 0$ are constants.

Lemma 2.3. (Sobolev-Poincare inequality [6]) Let's be a number with $2 \leq s<+\infty, n \leq 2 m$ and $2 \leq s<\frac{2 m}{n-2 m}, n>2 m$. Then there is a constant $k$ depending on $\Omega$ and $s$ such that 


$$
\|u\|_{s} \leq k\left\|(-\Delta)^{\frac{m}{2}} u\right\|, \forall u \in H_{0}^{m}(\Omega) .
$$

\section{Hausdorff Dimensions and Fractal Dimension for the Global Attractor}

\subsection{Differentiability of the Semigroup}

We denote $E_{0}=H^{m} \times H^{m} \times L^{2} \times L^{2}$. The inner product and the norm in $E_{0}$ space are defined as follows:

$$
\begin{gathered}
\forall \varphi_{i}=\left(u_{i}, v_{i}, p_{i}, q_{i}\right) \in E_{0}(i=1,2) \text {, we can get } \\
\begin{aligned}
\left(\varphi_{1}, \varphi_{2}\right)_{E_{0}}= & \left(\nabla^{m} u_{1}, \nabla^{m} u_{2}\right)+\left(\nabla^{m} v_{1}, \nabla^{m} v_{2}\right)+\left(p_{1}, p_{2}\right)+\left(q_{1}, q_{2}\right) . \\
& \left\|\varphi_{1}\right\|_{E_{0}}^{2}=\left(\varphi_{1}, \varphi_{1}\right)_{E_{0}} \\
& =\left\|\nabla^{m} u_{1}\right\|^{2}+\left\|\nabla^{m} v_{1}\right\|^{2}+\left\|p_{1}\right\|^{2}+\left\|q_{1}\right\|^{2} .
\end{aligned}
\end{gathered}
$$

Setting $\forall \varphi=(u, v, p, q)^{\mathrm{T}} \in E_{0}, p=u_{t}+\varepsilon u, q=v_{t}+\varepsilon v, \varepsilon>0$, the equations (1.1) and (1.2) are equivalent to

$$
\varphi_{t}+H(\varphi)=F(\varphi)
$$

where

$$
\begin{gathered}
H(\varphi)=\left(\begin{array}{c}
\varepsilon u-p \\
\varepsilon v-q \\
-\varepsilon p+\beta(-\Delta)^{m} p+\varepsilon^{2} u+(1-\varepsilon \beta)(-\Delta)^{m} u \\
-\varepsilon q+\beta(-\Delta)^{m} q+\varepsilon^{2} v+(1-\varepsilon \beta)(-\Delta)^{m} v
\end{array}\right), \\
0 \\
0 \\
F(\varphi)=\left(\begin{array}{c}
{ }^{m} u-g_{1}(u, v)+f_{1}(x) \\
\left.(I-M(v))(-\Delta)^{m}\right) \\
(I-M(v))(-\Delta)^{m} v-g_{2}(u, v)+f_{2}(x)
\end{array}\right) .
\end{gathered}
$$

Lemma 3.1.1. $\forall \varphi=(u, v, p, q)^{\mathrm{T}} \in E_{0}$, we can get

$$
(H(\varphi), \varphi)_{E_{0}} \geq \frac{\varepsilon}{4}\|\varphi\|_{E_{0}}^{2}+\frac{\beta}{4}\left\|\nabla^{m} p\right\|^{2}+\frac{\beta}{4}\left\|\nabla^{m} q\right\|^{2} .
$$

Proof. According to (3.1)-(3.5), Holder inequality, Young's inequality and Poincare inequality, we can obtain

$$
\begin{aligned}
& (H(\varphi), \varphi)_{E_{0}} \\
= & \left(\varepsilon \nabla^{m} u-\nabla^{m} p, \nabla^{m} u\right)+\left(\varepsilon \nabla^{m} v-\nabla^{m} q, \nabla^{m} v\right)+\left(-\varepsilon p+\beta(-\Delta)^{m} p\right. \\
& \left.+\varepsilon^{2} u+(1-\varepsilon \beta)(-\Delta)^{m} u, p\right)+\left(-\varepsilon q+\beta(-\Delta)^{m} q+\varepsilon^{2} v+(1-\varepsilon \beta)(-\Delta)^{m} v, q\right) \\
= & \varepsilon\left\|\nabla^{m} u\right\|^{2}+\varepsilon\left\|\nabla^{m} v\right\|^{2}-\varepsilon\|p\|^{2}-\varepsilon\|q\|^{2}+\beta\left\|\nabla^{m} p\right\|^{2}+\beta\left\|\nabla^{m} q\right\|^{2} \\
& +\varepsilon^{2}(u, p)+\varepsilon^{2}(v, q)-\varepsilon \beta\left(\nabla^{m} u, \nabla^{m} p\right)-\varepsilon \beta\left(\nabla^{m} v, \nabla^{m} q\right)
\end{aligned}
$$




$$
\begin{aligned}
\geq & \varepsilon\left\|\nabla^{m} u\right\|^{2}+\varepsilon\left\|\nabla^{m} v\right\|^{2}-\varepsilon\|p\|^{2}-\varepsilon\|q\|^{2}+\beta\left\|\nabla^{m} p\right\|^{2}+\beta\left\|\nabla^{m} q\right\|^{2} \\
& -\frac{\varepsilon^{2}}{2 \lambda_{1}^{m}}\left\|\nabla^{m} u\right\|^{2}-\frac{\varepsilon^{2}}{2}\|p\|^{2}-\frac{\varepsilon^{2}}{2 \lambda_{1}^{m}}\left\|\nabla^{m} v\right\|^{2}-\frac{\varepsilon^{2}}{2}\|q\|^{2}-\frac{\beta \varepsilon^{2}}{2}\left\|\nabla^{m} u\right\|^{2} \\
& -\frac{\beta}{2}\left\|\nabla^{m} p\right\|^{2}-\frac{\beta \varepsilon^{2}}{2}\left\|\nabla^{m} v\right\|^{2}-\frac{\beta}{2}\left\|\nabla^{m} q\right\|^{2} \\
\geq & \left(\varepsilon-\frac{\varepsilon^{2}}{2 \lambda_{1}^{m}}-\frac{\beta \varepsilon^{2}}{2}\right)\left\|\nabla^{m} u\right\|^{2}+\left(\varepsilon-\frac{\varepsilon^{2}}{2 \lambda_{1}^{m}}-\frac{\beta \varepsilon^{2}}{2}\right)\left\|\nabla^{m} v\right\|^{2}+\frac{\beta}{4}\left\|\nabla^{m} p\right\|^{2} \\
& +\frac{\beta}{4} \lambda_{1}^{m}\|p\|^{2}+\left(-\varepsilon-\frac{\beta \varepsilon^{2}}{2}\right)\|p\|^{2}+\frac{\beta}{4}\left\|\nabla^{m} q\right\|^{2}+\frac{\beta}{4} \lambda_{1}^{m}\|q\|^{2}+\left(-\varepsilon-\frac{\beta \varepsilon^{2}}{2}\right)\|q\|^{2} \\
\geq & \frac{\varepsilon}{4}\left(\left\|\nabla^{m} u\right\|^{2}+\left\|\nabla^{m} v\right\|^{2}+\|p\|^{2}+\|q\|^{2}\right)+\frac{\beta}{4}\left\|\nabla^{m} p\right\|^{2}+\frac{\beta}{4}\left\|\nabla^{m} q\right\|^{2} \\
\geq & \frac{\varepsilon}{4}\|\varphi\|_{E_{0}}^{2}+\frac{\beta}{4}\left\|\nabla^{m} p\right\|^{2}+\frac{\beta}{4}\left\|\nabla^{m} q\right\|^{2} .
\end{aligned}
$$

The proof of Lemma 3.1.1. is completed.

The linearized equations of (1.1)-(1.6), the above equations as follows:

$$
\begin{gathered}
U_{t t}+M(v)(-\Delta)^{m} U+2 M^{\prime}(v)(\nabla U, \nabla u)(-\Delta)^{m} u \\
+2 M^{\prime}(v)(\nabla V, \nabla v)(-\Delta)^{m} u+\beta(-\Delta)^{m} U_{t}+g_{1 u}(u, v) U+g_{1 v}(u, v) V=0, \\
V_{t t}+M(v)(-\Delta)^{m} V+2 M^{\prime}(v)(\nabla U, \nabla u)(-\Delta)^{m} v \\
+2 M^{\prime}(v)(\nabla V, \nabla v)(-\Delta)^{m} v+\beta(-\Delta)^{m} V_{t}+g_{2 u}(u, v) U+g_{2 v}(u, v) V=0, \\
\left.U(x, t)\right|_{x \in \partial \Omega}=0, U(x, 0)=\xi_{1}, U_{t}(x, 0)=\xi_{2}, \\
\left.V(x, t)\right|_{x \in \partial \Omega}=0, V(x, 0)=\eta_{1}, V_{t}(x, 0)=\eta_{2},
\end{gathered}
$$

where $\left(\xi_{1}, \eta_{1}, \xi_{2}, \eta_{2}\right) \in E_{0},\left(u, v, u_{t}, v_{t}\right)=S(t)\left(u_{0}, v_{0}, u_{1}, v_{1}\right)$ is the solution of (1.1)-(1.6) with $\left(u_{0}, v_{0}, u_{1}, v_{1}\right) \in \mathrm{A}$.

Given $\left(u_{0}, v_{0}, u_{1}, v_{1}\right) \in \mathrm{A}$ and $S(t): E_{0} \rightarrow E_{0}$, the solution $S(t)\left(u_{0}, v_{0}, u_{1}, v_{1}\right) \in E_{0}$ by stand methods we can show that for any $\left(\xi_{1}, \eta_{1}, \xi_{2}, \eta_{2}\right) \in E_{0}$, the linear initial boundary value problem (3.8)-(3.11) possess a unique solution:

$$
\left(U(t), V(t), U_{t}(t), V_{t}(t)\right) \in L^{\infty}\left((0,+\infty) ; E_{0}\right) .
$$

Lemma 3.1.2. For any $t>0, R>0$, the mapping $S(t): E_{0} \rightarrow E_{0}$ is Frechet differentiable on. It is differential at $\varphi_{0}=\left(u_{0}, v_{0}, u_{1}, v_{1}\right)$ is the linear operator on $F:\left(\xi_{1}, \eta_{1}, \xi_{2}, \eta_{2}\right)^{\mathrm{T}} \rightarrow(U(t), V(t), P(t), Q(t))^{\mathrm{T}}$, where $U(t)$ and $V(t)$ are solutions of (3.8)-(3.11)

Proof. Let $\varphi_{0}=\left(u_{0}, v_{0}, u_{1}, v_{1}\right)^{\mathrm{T}} \in E_{0}, \quad \tilde{\varphi}_{0}=\left(u_{0}+\xi_{1}, v_{0}+\eta_{1}, u_{1}+\xi_{2}, v_{1}+\eta_{2}\right)^{\mathrm{T}} \in E_{0}$ with $\|\varphi\|_{E_{0}} \leq R,\|\tilde{\varphi}\|_{E_{0}} \leq R$, we define $\left(u, v, u_{t}, v_{t}\right)=S(t) \varphi_{0},\left(\tilde{u}, \tilde{v}, \tilde{u}_{t}, \tilde{v}_{t}\right)=S(t) \tilde{\varphi}_{0}$. We can obtain the Lipchitz property of $S(t)$ on the bounded sets of $E_{0}$, that is

$$
\left\|S(t) \varphi_{0}-S(t) \tilde{\varphi}_{0}\right\|_{E_{0}}^{2} \leq \mathrm{e}^{C_{11} t}\left\|\left(\xi_{1}, \eta_{1}, \xi_{2}, \eta_{2}\right)\right\|_{E_{0}}^{2} .
$$

Let $\theta=\tilde{u}-u-U$ and $\sigma=\tilde{v}-v-V$ are solutions of problem 


$$
\begin{gathered}
\theta_{t t}+M(v) A^{m} \theta+\beta A^{m} \theta_{t}=h_{1}, \\
\sigma_{t t}+M(v) A^{m} \sigma+\beta A^{m} \sigma_{t}=h_{2}, \\
\theta(0)=\theta_{t}(0)=0, \\
\sigma(0)=\sigma_{t}(0)=0,
\end{gathered}
$$

with

$$
\begin{aligned}
h_{1}= & (M(v)-M(\tilde{v}))(-\Delta)^{m} \tilde{u}+2 M^{\prime}(v)(\nabla U, \nabla u)(-\Delta)^{m} u \\
& +2 M^{\prime}(v)(\nabla V, \nabla v)(-\Delta)^{m} u+g_{1}(u, v)-g_{1}(\tilde{u}, \tilde{v})+g_{1 u}(u, v) U+g_{1 v}(u, v) V, \\
h_{2}= & (M(v)-M(\tilde{v}))(-\Delta)^{m} \tilde{v}+2 M^{\prime}(v)(\nabla U, \nabla u)(-\Delta)^{m} v \\
& +2 M^{\prime}(v)(\nabla V, \nabla v)(-\Delta)^{m} v+g_{2}(u, v)-g_{2}(\tilde{u}, \tilde{v})+g_{2 u}(u, v) U+g_{2 v}(u, v) V,
\end{aligned}
$$

where $v=\|\nabla u\|^{2}+\|\nabla v\|^{2}, \tilde{v}=\|\nabla \tilde{u}\|^{2}+\|\nabla \tilde{v}\|^{2}$.

Taking the scalar product of each side of (3.14) with $\theta_{t}$. Because of

$$
\begin{gathered}
(\nabla u, \nabla u)=(\nabla \tilde{u}-\nabla u-\nabla \tilde{u},-\nabla u)=-(\nabla \tilde{u}-\nabla u, \nabla u)+(\nabla \tilde{u}, \nabla u) . \\
(\nabla \tilde{u}, \nabla \tilde{u})=(\nabla \tilde{u}-\nabla u+\nabla u, \nabla \tilde{u})=(\nabla \tilde{u}-\nabla u, \nabla \tilde{u})+(\nabla u, \nabla \tilde{u}) .
\end{gathered}
$$

So

$$
(\nabla u, \nabla u)-(\nabla \tilde{u}, \nabla \tilde{u})=(\nabla u-\nabla \tilde{u}, \nabla u+\nabla \tilde{u})
$$

$$
\begin{aligned}
&(M(v)-M(\tilde{v}))(-\Delta)^{m} \tilde{u}+2 M^{\prime}(v)(\nabla U, \nabla u)(-\Delta)^{m} u \\
&+\left.2 M^{\prime}(v)(\nabla V, \nabla v)(-\Delta)^{m} u, \theta_{t}\right) \\
&=\left(M^{\prime}(\theta v+(1-\theta) \tilde{v})(v-\tilde{v})(-\Delta)^{m} \tilde{u}+2 M^{\prime}(v)((\nabla U, \nabla u)+(\nabla V, \nabla v))(-\Delta)^{m} u, \theta_{t}\right) \\
&= M^{\prime}(\theta v+(1-\theta) \tilde{v})((\nabla u, \nabla u)-(\nabla \tilde{u}, \nabla \tilde{u})+(\nabla v, \nabla v)-(\nabla \tilde{v}, \nabla \tilde{v}))\left((-\Delta)^{m} \tilde{u}, \theta_{t}\right) \\
&+ 2 M^{\prime}(v)((\nabla U, \nabla u)+(\nabla V, \nabla v))\left((-\Delta)^{m} u, \theta_{t}\right) \\
&= M^{\prime}(\theta v+(1-\theta) \tilde{v})((\nabla \hat{u}, \nabla u+\nabla \tilde{u})+(\nabla \hat{v}, \nabla v+\nabla \tilde{v}))\left((-\Delta)^{m} \tilde{u}, \theta_{t}\right) \\
&+2 M^{\prime}(v)((\nabla U, \nabla u)+(\nabla V, \nabla v))\left((-\Delta)^{m} u, \theta_{t}\right) \\
&=\left[M^{\prime}(\theta v+(1-\theta) \tilde{v})((\nabla \hat{u}, \nabla u+\nabla \tilde{u})+(\nabla \hat{v}, \nabla v+\nabla \tilde{v}))\left((-\Delta)^{m} \tilde{u}, \theta_{t}\right)\right. \\
&\left.-M^{\prime}(v)((\nabla \hat{u}, \nabla u+\nabla \tilde{u})+(\nabla \hat{v}, \nabla v+\nabla \tilde{v}))\left((-\Delta)^{m} \tilde{u}, \theta_{t}\right)\right] \\
&+\left[M^{\prime}(v)((\nabla \hat{u}, \nabla u+\nabla \tilde{u})+(\nabla \hat{v}, \nabla v+\nabla \tilde{v}))\left((-\Delta)^{m} \tilde{u}, \theta_{t}\right)\right. \\
&\left.-2 M^{\prime}(v)((\nabla \hat{u}+\nabla \theta, \nabla u)+(\nabla \hat{v}+\nabla \sigma, \nabla v))\left((-\Delta)^{m} \tilde{u}, \theta_{t}\right)\right] \\
&+\left[-2 M^{\prime}(v)((\nabla U, \nabla u)+(\nabla V, \nabla v))\left((-\Delta)^{m} \tilde{u}, \theta_{t}\right)\right. \\
&\left.+2 M^{\prime}(v)((\nabla U, \nabla u)+(\nabla V, \nabla v))\left((-\Delta)^{m} u, \theta_{t}\right)\right] \\
&=I_{1}+I_{2}+I_{3},
\end{aligned}
$$




$$
\begin{aligned}
& I_{1}=\left(M^{\prime}(\theta v+(1-\theta) \tilde{v})-M^{\prime}(v)\right)((\nabla \hat{u}, \nabla u+\nabla \tilde{u})+(\nabla \hat{v}, \nabla v+\nabla \tilde{v}))\left((-\Delta)^{m} \tilde{u}, \theta_{t}\right) \\
& =M^{\prime \prime}(\zeta)(\tilde{v}-v)((\nabla \hat{u}, \nabla u+\nabla \tilde{u})+(\nabla \widehat{v}, \nabla v+\nabla \tilde{v}))\left((-\Delta)^{m} \tilde{u}, \theta_{t}\right) \\
& \leq M^{\prime \prime}(\zeta)(\|\nabla \hat{u}\|\|\nabla u+\nabla \tilde{u}\|+\|\nabla \widehat{v}\|\|\nabla v+\nabla \tilde{v}\|)^{2}\left\|\nabla^{m} \tilde{u}\right\|\left\|\nabla^{m} \theta_{t}\right\| \\
& \leq 2 M^{\prime \prime}(\zeta)\left(\|\nabla \hat{u}\|^{2}\|\nabla u+\nabla \tilde{u}\|^{2}+\|\nabla \hat{v}\|^{2}\|\nabla v+\nabla \tilde{v}\|^{2}\right)\left\|\nabla^{m} \tilde{u}\right\|\left\|\nabla^{m} \theta_{t}\right\| \\
& \leq 2 \lambda_{1}^{-m+1} M^{\prime \prime}(\zeta)\left(\left\|\nabla^{m} \hat{u}\right\|^{2}(\|\nabla u\|+\|\nabla \tilde{u}\|)^{2}+\left\|\nabla^{m} \hat{v}\right\|^{2}(\|\nabla v\|+\|\nabla \tilde{v}\|)^{2}\right)\left\|\nabla^{m} \tilde{u}\right\|\left\|\nabla^{m} \theta_{t}\right\|, \\
& I_{2}=M^{\prime}(v)((\nabla \hat{u}, \nabla u+\nabla \tilde{u})+(\nabla \widehat{v}, \nabla v+\nabla \tilde{v}))\left((-\Delta)^{m} \tilde{u}, \theta_{t}\right) \\
& -2 M^{\prime}(v)((\nabla \hat{u}+\nabla \theta, \nabla u)+(\nabla \widehat{v}+\nabla \sigma, \nabla v))\left((-\Delta)^{m} \tilde{u}, \theta_{t}\right) \\
& =M^{\prime}(v)((\nabla \hat{u}, \nabla \widehat{u})+(\nabla \widehat{v}, \nabla \widehat{v}))\left((-\Delta)^{m} \tilde{u}, \theta_{t}\right) \\
& -2 M^{\prime}(v)((\nabla \theta, \nabla u)+(\nabla \sigma, \nabla v))\left((-\Delta)^{m} \tilde{u}, \theta_{t}\right) \\
& \leq M^{\prime}(v)\left(\|\nabla \hat{u}\|^{2}+\|\nabla \hat{v}\|^{2}\right)\left\|\nabla^{m} \tilde{u}\right\|\left\|\nabla^{m} \theta_{t}\right\| \\
& -2 M^{\prime}(v)((\nabla \theta, \nabla u)+(\nabla \sigma, \nabla v))\left((-\Delta)^{m} \tilde{u}, \theta_{t}\right) \\
& \leq M^{\prime}(v)\left(\|\nabla \hat{u}\|^{2}+\|\nabla \widehat{v}\|^{2}\right)\left\|\nabla^{m} \tilde{u}\right\|\left\|\nabla^{m} \theta_{t}\right\| \\
& +2 \lambda_{1}{ }^{\frac{-m+1}{2}} M^{\prime}(v)\left(\left\|\nabla^{m} \theta\right\|\|\nabla u\|+\left\|\nabla^{m} \sigma\right\|\|\nabla v\|\right)\left\|\nabla^{m} \tilde{u}\right\|\left\|\nabla^{m} \theta_{t}\right\| \text {, } \\
& I_{3}=2 M^{\prime}(v)((\nabla U, \nabla u)+(\nabla V, \nabla v))\left((-\Delta)^{m} \widehat{u}, \theta_{t}\right) \\
& =2 M^{\prime}(v)((\nabla \tilde{u}-\nabla u-\nabla \theta, \nabla u)+(\nabla \tilde{v}-\nabla v-\nabla \sigma, \nabla v))\left(\nabla^{m} \widehat{u}, \nabla^{m} \theta_{t}\right) \\
& \leq 2 M^{\prime}(v)(\|\nabla \hat{u}\|\|\nabla u\|+\|\nabla \theta\|\|\nabla u\|+\|\nabla \widehat{v}\|\|\nabla v\|+\|\nabla \sigma\|\|\nabla v\|)\left\|\nabla^{m} \widehat{u}\right\|\left\|\nabla^{m} \theta_{t}\right\| \\
& \leq 2 \lambda_{1}^{\frac{-m+1}{2}} M^{\prime}(v)\left(\left\|\nabla^{m} \hat{u}\right\|\|\nabla u\|+\left\|\nabla^{m} \theta\right\|\|\nabla u\|+\left\|\nabla^{m} \widehat{v}\right\|\|\nabla v\|+\left\|\nabla^{m} \sigma\right\|\|\nabla v\|\right)\left\|\nabla^{m} \hat{u}\right\|\left\|\nabla^{m} \theta_{t}\right\| \\
& \leq 2 \lambda_{1}^{\frac{-m+1}{2}} M^{\prime}(v)\left(\left\|\nabla^{m} \hat{u}\right\|^{2}\|\nabla u\|\left\|\nabla^{m} \theta_{t}\right\|+\left\|\nabla^{m} \theta\right\|\|\nabla u\|\left(\left\|\nabla^{m} u\right\|+\left\|\nabla^{m} \tilde{u}\right\|\right)\left\|\nabla^{m} \theta_{t}\right\|\right. \\
& \left.+\left(\left\|\nabla^{m} \hat{v}\right\|^{2}+\left\|\nabla^{m} \hat{u}\right\|^{2}\right)\|\nabla v\|\left\|\nabla^{m} \theta_{t}\right\|+\left\|\nabla^{m} \sigma\right\|\|\nabla v\|\left(\left\|\nabla^{m} u\right\|+\left\|\nabla^{m} \tilde{u}\right\|\right)\left\|\nabla^{m} \theta_{t}\right\|\right),
\end{aligned}
$$

where $\hat{u}=u-\tilde{u}, \widehat{v}=v-\tilde{v}$.

By (H3) and Young's inequality, we can get

$$
\begin{aligned}
& \left(g_{1}(u, v)-g_{1}(\tilde{u}, \tilde{v})+g_{1 u}(u, v) U+g_{1 v}(u, v) V, \theta_{t}\right) \\
& =\left(g_{1}(u, v)-g_{1}(\tilde{u}, \tilde{v})+g_{1 u}(u, v)(\tilde{u}-u-\theta)+g_{1 v}(u, v)(\tilde{v}-v-\sigma), \theta_{t}\right) \\
& =\left(g_{1}(u, v)-g_{1}(\tilde{u}, \tilde{v})+g_{1 u}(u, v) \hat{u}-g_{1 u}(u, v) \theta+g_{1 v}(u, v) \hat{v}-g_{1 v}(u, v) \sigma, \theta_{t}\right) \\
& =\left(g_{1}(u, v)-g_{1}(\tilde{u}, v)+g_{1}(\tilde{u}, v)-g_{1}(\tilde{u}, \tilde{v})+g_{1 u}(u, v) \hat{u}+g_{1 v}(u, v) \hat{v}, \theta_{t}\right) \\
& +\left(-g_{1 u}(u, v) \theta-g_{1 v}(u, v) \sigma, \theta_{t}\right)
\end{aligned}
$$




$$
\begin{aligned}
= & \left(\left(g_{1 u}(u, v)-g_{1 u}(\xi, v)\right) \hat{u}+\left(g_{1 v}(u, v)-g_{1 v}(\tilde{u}, \eta)\right) \hat{v}, \theta_{t}\right) \\
& +\left(-g_{1 u}(u, v) \theta-g_{1 v}(u, v) \sigma, \theta_{t}\right) \\
\leq & \left\|g_{1 u}(u, v)-g_{1 u}(\xi, v)\right\|_{L^{\infty}}\|\hat{u}\|\left\|\theta_{t}\right\|+\left\|g_{1 v}(u, v)-g_{1 v}(\tilde{u}, \eta)\right\|_{L^{\infty}}\|v\|\left\|\theta_{t}\right\| \\
& +\left\|g_{1 u}(u, v)\right\|_{L^{\infty}}\|\theta\|\left\|\theta_{t}\right\|+\left\|g_{1 v}(u, v)\right\|_{L^{\infty}}\|\sigma\|\left\|\theta_{t}\right\| \\
\leq & k_{1}\|\tilde{u}\|^{k+1}\left\|\theta_{t}\right\|+k_{2}\left(\|\tilde{u}\|^{k+1}(\|v\|+\|\tilde{v}\|)+\|\hat{v}\|^{k+1}\right)\left\|\theta_{t}\right\|+C_{16}\|\theta\|^{2}+\frac{2}{4}\left\|\theta_{t}\right\|^{2}+C_{17}\|\sigma\|^{2} .
\end{aligned}
$$

By using Young inequality, we can get

$$
\begin{aligned}
h_{1} \leq & \frac{C_{1}}{\varepsilon}\left\|\nabla^{m} u-\nabla^{m} \tilde{u}\right\|^{4}+\frac{C_{2}}{\varepsilon}\left\|\nabla^{m} v-\nabla^{m} \tilde{v}\right\|^{4}+\frac{C_{3}}{\varepsilon}\left\|\nabla^{m} \theta\right\|^{2}+\frac{C_{4}}{\varepsilon}\left\|\nabla^{m} \sigma\right\|^{2} \\
& +\frac{11 \varepsilon}{4}\left\|\nabla^{m} \theta_{t}\right\|^{2}+C_{5}\left\|\nabla^{m} \tilde{u}\right\|^{2 k+2}+\frac{5}{4}\left\|\theta_{t}\right\|^{2}+C_{6}\left\|\nabla^{m} \vec{v}\right\|^{2 k+2}+C_{7}\|\theta\|^{2}+C_{8}\|\sigma\|^{2} .
\end{aligned}
$$

From (3.23)-(3.28), we have

$$
\begin{aligned}
\frac{\mathrm{d}}{\mathrm{d} t}\left\|\theta_{t}\right\|^{2}+M(v) \frac{\mathrm{d}}{\mathrm{d} t}\left\|\nabla^{m} \theta\right\|^{2} \leq & C_{9}\left(\left\|\nabla^{m} \widehat{u}\right\|^{4}+\left\|\nabla^{m} \theta\right\|^{2}+\left\|\nabla^{m} \hat{v}\right\|^{4}+\left\|\nabla^{m} \sigma\right\|^{2}\right) \\
& +C_{10}\left(\left\|\nabla^{m} \widehat{u}\right\|^{2 k+2}+\left\|\nabla^{m} \widehat{v}\right\|^{2 k+2}+\left\|\theta_{t}\right\|^{2}\right) .
\end{aligned}
$$

Take the scalar product of each side of (3.15) with $\sigma_{t}$. Because of

$$
\begin{aligned}
\frac{\mathrm{d}}{\mathrm{d} t}\left\|\sigma_{t}\right\|^{2}+M(v) \frac{\mathrm{d}}{\mathrm{d} t}\left\|\nabla^{m} \sigma\right\|^{2} \leq & C_{11}\left(\left\|\nabla^{m} \hat{u}\right\|^{4}+\left\|\nabla^{m} \theta\right\|^{2}+\left\|\nabla^{m} \vec{v}\right\|^{4}+\left\|\nabla^{m} \sigma\right\|^{2}\right) \\
& +C_{12}\left(\left\|\nabla^{m} \widehat{u}\right\|^{2 k+2}+\left\|\nabla^{m} \hat{v}\right\|^{2 k+2}+\left\|\theta_{t}\right\|^{2}\right) .
\end{aligned}
$$

Summing up (3.29) and (3.30), we have

$$
\begin{aligned}
& \frac{\mathrm{d}}{\mathrm{d} t}\left(\left\|\theta_{t}\right\|^{2}+\left\|\sigma_{t}\right\|^{2}+\mu\left(\left\|\nabla^{m} \theta\right\|^{2}+\left\|\nabla^{m} \sigma\right\|^{2}\right)\right) \\
& \leq C_{13}\left(\left\|\theta_{t}\right\|^{2}+\left\|\sigma_{t}\right\|^{2}+\mu\left(\left\|\nabla^{m} \theta\right\|^{2}+\left\|\nabla^{m} \sigma\right\|^{2}\right)\right) \\
& +C_{14}\left(\left\|\nabla^{m} \hat{u}\right\|^{4}+\left\|\nabla^{m} \vec{v}\right\|^{4}+\left\|\nabla^{m} \vec{u}\right\|^{2 k+2}+\left\|\nabla^{m} \widehat{v}\right\|^{2 k+2}\right) .
\end{aligned}
$$

By using Gronwall inequality, we can get

$$
\begin{aligned}
\left\|\theta_{t}\right\|^{2}+\left\|\sigma_{t}\right\|^{2}+\mu\left(\left\|\nabla^{m} \theta\right\|^{2}+\left\|\nabla^{m} \sigma\right\|^{2}\right) \\
\leq C_{15} \mathrm{e}^{C_{16} t} \int_{0}^{t}\left(\left\|\nabla^{m} \hat{u}\right\|^{4}+\left\|\nabla^{m} \widehat{v}\right\|^{4}+\left\|\nabla^{m} \widehat{u}\right\|^{2 k+2}+\left\|\nabla^{m} \widehat{v}\right\|^{2 k+2}\right) \mathrm{d} \tau \\
\leq C_{17} \mathrm{e}^{C_{10^{t}}}\left[\left(\left\|\nabla^{m} \xi_{1}\right\|^{2}+\left\|\nabla^{m} \eta_{1}\right\|^{2}+\left\|\xi_{2}\right\|^{2}+\left\|\eta_{2}\right\|^{2}\right)^{2}\right. \\
\left.+\left(\left\|\nabla^{m} \xi_{1}\right\|^{2}+\left\|\nabla^{m} \eta_{1}\right\|^{2}+\left\|\xi_{2}\right\|^{2}+\left\|\eta_{2}\right\|^{2}\right)^{k+1}\right] \\
\leq C_{20} \mathrm{e}^{C_{19 t} t}\left(\left\|\left(\xi_{1}, \eta_{1}, \xi_{2}, \eta_{2}\right)\right\|_{E_{0}}^{4}+\left\|\left(\xi_{1}, \eta_{1}, \xi_{2}, \eta_{2}\right)\right\|_{E_{0}}^{2 k+2}\right),
\end{aligned}
$$

where $C_{17}, C_{18}, C_{19}, C_{20}>0$.

From (3.32), we can get 


$$
\begin{aligned}
& \frac{\|\tilde{\varphi}(t)-\varphi(t)-B(t)\|_{E_{0}}^{2}}{\left\|\left(\xi_{1}, \eta_{1}, \xi_{2}, \eta_{2}\right)^{\mathrm{T}}\right\|_{E_{0}}^{2}} \\
& \leq \frac{C_{20} \mathrm{e}^{C_{19} t}\left(\left\|\left(\xi_{1}, \eta_{1}, \xi_{2}, \eta_{2}\right)^{\mathrm{T}}\right\|_{E_{0}}^{4}+\left\|\left(\xi_{1}, \eta_{1}, \xi_{2}, \eta_{2}\right)^{\mathrm{T}}\right\|_{E_{0}}^{2 k+2}\right)}{\left\|\left(\xi_{1}, \eta_{1}, \xi_{2}, \eta_{2}\right)^{\mathrm{T}}\right\|_{E_{0}}^{2}} \\
& \leq C_{20} \mathrm{e}^{C_{19 t}}\left(\left\|\left(\xi_{1}, \eta_{1}, \xi_{2}, \eta_{2}\right)^{\mathrm{T}}\right\|_{E_{0}}^{2}+\left\|\left(\xi_{1}, \eta_{1}, \xi_{2}, \eta_{2}\right)^{\mathrm{T}}\right\|_{E_{0}}^{2 k}\right) \rightarrow 0,
\end{aligned}
$$

here

$$
B(t)=\left(U(t), V(t), U_{t}(t), V_{t}(t)\right) .
$$

As $\left(\xi_{1}, \eta_{1}, \xi_{2}, \eta_{2}\right)^{\mathrm{T}} \rightarrow 0$ in $E_{0}$. The lemma 3.1.2 is completed.

\subsection{The Upper Bounds of Hausdorff Dimension and Fractal Dimension for the Global Attractor}

Consider the first variation of (3.3) with initial condition;

$$
\Psi_{t}^{\prime}+p(\varphi) \Psi=\Gamma_{1}(\varphi) \Psi+\Gamma_{2} \Psi^{\mathrm{T}}, \Psi(0)=\left(\xi_{1}, \eta_{1}, \xi_{2}, \eta_{2}\right)^{\mathrm{T}} \in E_{0}, t>0,
$$

where $\Psi=(U, V, P, Q)^{\mathrm{T}} \in E_{0}, P=U_{t}+\varepsilon U, Q=V_{t}+\varepsilon V$ and $\varphi=(u, v, p, q)^{\mathrm{T}} \in E_{0}$ are solutions of (3.35),

$$
\begin{gathered}
p(\varphi)=\left(\begin{array}{cccc}
\varepsilon I & 0 & -I & 0 \\
0 & \varepsilon I & 0 & -I \\
\zeta_{1} & 0 & \zeta_{2} & 0 \\
0 & \zeta_{1} & 0 & \zeta_{2}
\end{array}\right), \\
\Gamma_{1}=\left(\begin{array}{cccc}
0 & 0 & 0 & 0 \\
0 & 0 & 0 & 0 \\
-g_{1 u}(u, v) & -g_{1 v}(u, v) & 0 & 0 \\
-g_{2 u}(u, v) & -g_{2 u}(u, v) & 0 & 0
\end{array}\right) . \\
\Gamma_{2}=\left(\begin{array}{llll}
0 & 0 & \zeta_{3} & \zeta_{4}
\end{array}\right)^{\mathrm{T}},
\end{gathered}
$$

where $\zeta_{1}=\varepsilon^{2}+(I-\beta \varepsilon)(-\Delta)^{m}, \zeta_{2}=-\varepsilon I+\beta(-\Delta)^{m}$,

$\zeta_{3}=[1-M(v)](-\Delta)^{m} U-2 M^{\prime}(v)(\nabla U, \nabla u)(-\Delta)^{m} u-2 M^{\prime}(v)(\nabla V, \nabla v)(-\Delta)^{m} u$,

$\zeta_{4}=[1-M(v)](-\Delta)^{m} V-2 M^{\prime}(v)(\nabla U, \nabla u)(-\Delta)^{m} v-2 M^{\prime}(v)(\nabla V, \nabla v)(-\Delta)^{m} v$.

It is easy to show from lemma 3.1.2. that (3.35) is a well-posed problem in $E_{0}$, the mapping $S_{\varepsilon}(\tau):\left\{u_{0}, v_{0}, p_{1}, q_{1}\right\} \rightarrow\{u, v, p, q\}$, where $p_{1}=u_{1}+\varepsilon u_{0}$, $q_{1}=v_{1}+\varepsilon v_{0}$,

$p=u_{t}+\varepsilon u, q=v_{t}+\varepsilon v$.

$\varphi(\tau)=\left\{u(\tau), v(\tau), p(\tau)=u_{t}(\tau)+\varepsilon u(\tau), q(\tau)=v_{t}(\tau)+\varepsilon v(\tau)\right\}$ is Frechet differentiable on $E_{0}$ for any $t>0$, its differential at $\varphi_{0}=\left\{u_{0}, v_{0}, p_{1}, q_{1}\right\}^{\mathrm{T}}$ is the linear operator on $E_{0},\left(\xi_{1}, \eta_{1}, \xi_{2}, \eta_{2}\right)^{\mathrm{T}} \rightarrow(U(t), V(t), P(t), Q(t))^{\mathrm{T}}$, where $(U(t), V(t), P(t), Q(t))^{\mathrm{T}}$ is the solution of (3.35). 
Lemma 3.2.1. For any orthonormal family of elements of $\left(E_{0},\|\|_{E_{0}}\right)$, $\left(\xi_{1 j}, \xi_{2 j}\right)^{\mathrm{T}},\left(\eta_{1 j}, \eta_{2 j}\right)^{\mathrm{T}}, j=1,2, \cdots, n_{1}$, we have

$$
\begin{aligned}
& \sum_{j=1}^{n_{1}}\left\|A^{\frac{1}{2} v} \xi_{1 j}\right\|^{2} \leq 2 \sum_{j=1}^{n_{1}} \mu_{j}^{v-1}, v \in[0,1), \\
& \sum_{j=1}^{n_{1}}\left\|A^{\frac{1}{2} v} \eta_{1 j}\right\|^{2} \leq 2 \sum_{j=1}^{n_{1}} \mu_{j}^{v-1}, v \in[0,1),
\end{aligned}
$$

where $\left\{\mu_{j}\right\}_{j=1}^{+\infty}$ is the eigenvalue of $(-\Delta)^{m}$.

Proof. This is a direct consequence of lemma VI 6.3 of [7]

Theorem 3.2.2. If we take proper $\mu_{0}, s$ satisfy $\frac{1-\mu_{0}}{2}+2 K \lambda_{1}^{-m+1} R_{1}^{2} \leq \frac{\varepsilon}{8}$ and (H1)-(H3) hold, then there exists $\rho\left(R_{1}\right)>0$, such that the Hausdorff dimension and Fractal dimension of global attractor $\mathrm{A}$ in $E_{0}$ satisfies

$$
\begin{gathered}
d_{H}(\mathrm{~A}) \leq \min \\
\left\{n_{1} \mid n_{1} \in N, \frac{1}{n_{1}} \sum_{j=1}^{n_{1}} \mu_{j}^{\delta-1}<\frac{\varepsilon}{4 \rho}\right\}, \\
d_{F}(\mathrm{~A}) \leq 2 n_{1},
\end{gathered}
$$

where $R_{0}$ is as in [1], and

$$
\delta=\left\{\begin{array}{l}
\frac{(n-2)(p-1)-2}{2}, \frac{n}{n-2 m} \leq r_{i} \leq \frac{n+2 m}{n-2 m}, n \geq 2 m, \\
0, n<2 m \text { or } 0 \leq r_{i} \leq \frac{n}{n-2 m}, n \geq 2 m,
\end{array}\right.
$$

here $i=1,2$.

Proof. Let $n_{1} \in N$ be fixed. Consider $n_{1}$ solutions $\Psi_{1}, \Psi_{2}, \cdots, \Psi_{n_{1}}$ of (3.35). At given time $\tau$, let $Q_{n_{1}}(\tau)$ define the orthogonal projection in $E_{0}$ onto $\operatorname{span}\left\{\Psi_{1}, \Psi_{2}, \cdots, \Psi_{n_{1}}\right\}$. Let $y_{j}(s)=\left(\xi_{1 j}, \eta_{1 j}, \xi_{2 j}, \eta_{2 j}\right)^{\mathrm{T}} \in E_{0}, j=1,2, \cdots, n_{1}$ be an Orthonormal basis of

$$
Q_{n_{1}} E_{0}=\operatorname{span}\left\{\Psi_{1}, \Psi_{2}, \cdots, \Psi_{n_{1}}\right\},
$$

with respect to the inner product $(,)_{E_{0}}$ and norm $\|\cdot\|_{E_{0}}$.

Suppose

$$
\varphi(\tau)=(u(\tau), v(\tau), p(\tau), q(\tau))^{\mathrm{T}} \in \mathrm{A},
$$

then $\|\varphi(\tau)\|_{E_{0}} \leq M_{0}, \forall s>\tau$. By $\left\|y_{j}\right\|_{E_{0}}=1$ and Lemma 4.1.1, we can get

$$
\begin{aligned}
& \quad-\left(p(\varphi(s)) y_{j}(s), y_{j}(s)\right)_{E_{0}} \leq-\frac{\varepsilon}{4}-\frac{\beta}{4}\left\|\nabla^{m} \xi_{2 j}\right\|^{2}-\frac{\beta}{4}\left\|\nabla^{m} \eta_{2 j}\right\|^{2} \\
& \left(\Gamma_{1}(\varphi(s)) y_{j}(s), y_{j}(s)\right)_{E_{0}} \\
& =\left(-g_{1 u}(u, v) \xi_{1 j}-g_{1 v}(u, v) \eta_{1 j}, \xi_{2 j}\right)+\left(-g_{2 u}(u, v) \xi_{1 j}-g_{2 v}(u, v) \eta_{1 j}, \eta_{2 j}\right) \\
& =\left(-g_{1 u}(u, v) \xi_{1 j}, \xi_{2 j}\right)+\left(-g_{1 v}(u, v) \eta_{1 j}, \xi_{2 j}\right) \\
& +\left(-g_{2 u}(u, v) \xi_{1 j}, \eta_{2 j}\right)+\left(-g_{2 v}(u, v) \eta_{1 j}, \eta_{2 j}\right)
\end{aligned}
$$




$$
\begin{aligned}
& \leq\left\|A^{-\frac{m}{2}}\left(g_{1 u}(u, v) \xi_{1 j}\right)\right\|\left\|\nabla^{m} \xi_{2 j}\right\|+\left\|A^{-\frac{m}{2}}\left(g_{1 v}(u, v) \eta_{1 j}\right)\right\|\left\|\nabla^{m} \xi_{2 j}\right\| \\
& +\left\|A^{-\frac{m}{2}}\left(g_{2 u}(u, v) \xi_{1 j}\right)\right\|\left\|\nabla^{m} \eta_{2 j}\right\|+\left\|A^{-\frac{m}{2}}\left(g_{2 u}(u, v) \eta_{1 j}\right)\right\|\left\|\nabla^{m} \eta_{2 j}\right\| .
\end{aligned}
$$

where $A=-\Delta$.

By the hypothesis (H4) in [1], the mean value theorem and Sobolev embedding theorem:

$$
H_{0}^{m \kappa}(\Omega) \subset D\left(A^{\frac{m}{2} \kappa}\right) \subset H^{m \kappa}(\Omega) \subset L^{q}(\Omega) \subset L^{2}(\Omega) \subset L^{q^{\prime}}(\Omega) \subset H^{-m \kappa}(\Omega),
$$

where $\frac{1}{q}=\frac{1}{2}-\frac{m \kappa}{n}, \frac{1}{q}+\frac{1}{q^{\prime}}=1, \kappa \in[0,1)$.

Thus, by Lemma 2.4. in [1] and (3.47), for $n=1$,

$$
H_{0}^{m \kappa}(\Omega) \subset L^{\infty}(\Omega) \subset L^{1}(\Omega) \subset H^{-m}(\Omega) \subset\left(H_{0}^{m}(\Omega)\right)^{\prime} .
$$

There exists $C_{21}\left(R_{0}\right), C_{23}\left(R_{0}\right), C_{25}\left(R_{0}\right), C_{27}\left(R_{0}\right)>0$, such that

$$
\begin{aligned}
& \left\|A^{-\frac{m}{2}}\left(g_{1 u}(u, v) \xi_{1 j}\right)\right\| \leq C_{21}\left|g_{1 u}(u, v) \xi_{1 j}\right|_{L^{1}} \leq C_{22}\left(R_{0}\right)\left\|\xi_{1 j}\right\|, \\
& \left\|A^{-\frac{m}{2}}\left(g_{1 v}(u, v) \eta_{1 j}\right)\right\| \leq C_{23}\left|g_{1 v}(u, v) \eta_{1 j}\right|_{L^{1}} \leq C_{24}\left(R_{0}\right)\left\|\eta_{1 j}\right\|, \\
& \left\|A^{-\frac{m}{2}}\left(g_{2 u}(u, v) \xi_{1 j}\right)\right\| \leq C_{25}\left|g_{2 u}(u, v) \xi_{1 j}\right|_{L^{1}} \leq C_{26}\left(R_{0}\right)\left\|\xi_{1 j}\right\|, \\
& \left\|A^{-\frac{m}{2}}\left(g_{2 v}(u, v) \eta_{1 j}\right)\right\| \leq C_{27}\left|g_{2 v}(u, v) \eta_{1 j}\right|_{L^{1}} \leq C_{28}\left(R_{0}\right)\left\|\eta_{1 j}\right\| .
\end{aligned}
$$

For $1<n<2 m$, by $H_{0}^{m}(\Omega) \subset L^{q}(\Omega) \subset H^{-m}(\Omega) \subset\left(H_{0}^{m}(\Omega)\right)^{\prime}, q>0$, there exists $C_{30}\left(R_{0}\right), C_{32}\left(R_{0}\right), C_{34}\left(R_{0}\right), C_{36}\left(R_{0}\right)>0$, such that

$$
\begin{aligned}
& \left\|A^{-\frac{m}{2}}\left(g_{1 u}(u, v) \xi_{1 j}\right)\right\| \leq C_{29}\left\|g_{1 u}(u, v) \xi_{1 j}\right\|_{L^{2}} \leq C_{30}\left(R_{0}\right)\left\|\xi_{1 j}\right\|, \\
& \left\|A^{-\frac{m}{2}}\left(g_{1 v}(u, v) \eta_{1 j}\right)\right\| \leq C_{31}\left\|g_{1 v}(u, v) \eta_{1 j}\right\|_{L^{\frac{2}{2}}} \leq C_{32}\left(R_{0}\right)\left\|\eta_{1 j}\right\|, \\
& \left\|A^{-\frac{m}{2}}\left(g_{2 u}(u, v) \xi_{1 j}\right)\right\| \leq C_{33}\left\|g_{2 u}(u, v) \xi_{1 j}\right\|_{L^{2}} \leq C_{34}\left(R_{0}\right)\left\|\xi_{1 j}\right\|, \\
& \left\|A^{-\frac{m}{2}}\left(g_{2 v}(u, v) \eta_{1 j}\right)\right\| \leq C_{35}\left\|g_{2 v}(u, v) \eta_{1 j}\right\|_{L^{2}} \leq C_{36}\left(R_{0}\right)\left\|\eta_{1 j}\right\| .
\end{aligned}
$$

For $n>2 m$, by (H4) in [1] there exists $C_{37}\left(R_{1}\right), C_{38}\left(R_{1}\right), C_{39}\left(R_{1}\right), C_{40}\left(R_{1}\right)>0$, such that

$$
\left\|A^{-\frac{m}{2}}\left(g_{1 u}(u, v) \xi_{1 j}\right)\right\| \leq\left\|g_{1 u}(u, v) \xi_{1 j}\right\|_{L^{n+2 m}} \leq C_{37}\left(R_{0}\right)\left\|A^{\frac{m}{2} \delta} \xi_{1 j}\right\|,
$$




$$
\begin{aligned}
& \left\|A^{-\frac{m}{2}}\left(g_{1 v}(u, v) \eta_{1 j}\right)\right\| \leq\left\|g_{1 v}(u, v) \eta_{1 j}\right\|_{L^{n+2 m}} \leq C_{38}\left(R_{0}\right)\left\|A^{\frac{m}{2} \delta} \eta_{1 j}\right\|, \\
& \left\|A^{-\frac{m}{2}}\left(g_{2 u}(u, v) \xi_{1 j}\right)\right\| \leq\left\|g_{2 u}(u, v) \xi_{1 j}\right\|_{L^{n+2 m}} \leq C_{39}\left(R_{0}\right)\left\|A^{\frac{m}{2} \delta} \xi_{1 j}\right\|, \\
& \left\|A^{-\frac{m}{2}}\left(g_{2 v}(u, v) \eta_{1 j}\right)\right\| \leq\left\|g_{2 v}(u, v) \eta_{1 j}\right\|_{L^{n+2 m}} \leq C_{40}\left(R_{0}\right)\left\|A^{\frac{m}{2} \delta} \eta_{1 j}\right\| .
\end{aligned}
$$

From above, we have

$$
\begin{aligned}
& \left(\Gamma_{1}(\varphi(s)) y_{j}(s), y_{j}(s)\right)_{E_{0}} \\
\leq & \frac{C_{41}}{2}\left\|A^{\frac{m}{2} \delta} \xi_{1 j}\right\|\left\|A^{\frac{m}{2}} \xi_{2 j}\right\|+\frac{C_{42}}{2}\left\|A^{\frac{m}{2} \delta} \eta_{1 j}\right\|\left\|A^{\frac{m}{2}} \xi_{2 j}\right\| \\
& +\frac{C_{43}}{2}\left\|A^{\frac{m}{2} \delta} \xi_{1 j}\right\|\left\|A^{\frac{m}{2}} \eta_{2 j}\right\|+\frac{C_{44}}{2}\left\|A^{\frac{m}{2} \delta} \eta_{1 j}\right\|\left\|A^{\frac{m}{2}} \eta_{2 j}\right\| \\
\leq & C_{45}\left(\left\|A^{\frac{m}{2} \delta} \xi_{1 j}\right\|\left\|A^{\frac{m}{2}} \xi_{2 j}\right\|+\left\|A^{\frac{m}{2} \delta} \eta_{1 j}\right\|\left\|A^{\frac{m}{2}} \xi_{2 j}\right\|\right. \\
& \left.+\left\|A^{\frac{m}{2} \delta} \xi_{1 j}\right\|\left\|A^{\frac{m}{2}} \eta_{2 j}\right\|+\left\|A^{\frac{m}{2} \delta} \eta_{1 j}\right\|\left\|A^{\frac{m}{2}} \eta_{2 j}\right\|\right),
\end{aligned}
$$

where $C_{41}=\max \left\{C_{22}, C_{37}\right\}, C_{42}=\max \left\{C_{24}, C_{38}\right\}, C_{43}=\max \left\{C_{26}, C_{39}\right\}$, $C_{44}=\max \left\{C_{28}, C_{40}\right\}$,

$$
C_{45}=\max \left\{C_{41}, C_{42}, C_{43}, C_{44}\right\} .
$$

According to Lemma 2.4. in [1], we can get

$$
\begin{aligned}
& \left.\Gamma_{2}(\varphi(s)) y_{j}(s), y_{j}(s)\right) \\
= & (1-M(v))\left(\nabla^{m} \xi_{1 j}, \nabla^{m} \xi_{2 j}\right)-2 M^{\prime}(v)\left(\left(\nabla \xi_{1 j}, \nabla u\right)+\left(\nabla \eta_{1 j}, \nabla v\right)\right)\left(\nabla^{m} u, \nabla^{m} \xi_{2 j}\right) \\
& +(1-M(v)) \lambda_{j}^{\frac{m}{2}}\left(\nabla^{m} \eta_{1 j}, \nabla^{m} \eta_{2 j}\right) \\
& -2 M^{\prime}(v) \lambda_{j}^{\frac{m}{2}}\left(\left(\nabla \xi_{1 j}, \nabla u\right)+\left(\nabla \eta_{1 j}, \nabla v\right)\right)\left(\nabla^{m} v, \nabla^{m} \eta_{2 j}\right) \\
\leq & \left(1-\mu_{0}\right) \lambda_{j}^{\frac{m}{2}}\left\|\nabla^{m} \xi_{1 j}\right\|\left\|\xi_{2 j}\right\|+2 \lambda_{1}^{-m+1} R_{0}^{2} M^{\prime}(v) \lambda_{j}^{\frac{m}{2}}\left(\left\|\nabla^{m} \xi_{1 j}\right\|\left\|\xi_{2 j}\right\|+\left\|\nabla^{m} \eta_{1 j}\right\|\left\|\xi_{2 j}\right\|\right) \\
& +\left(1-\mu_{0}\right) \lambda_{j}^{\frac{m}{2}}\left\|\nabla^{m} \eta_{1 j}\right\|\left\|\eta_{2 j}\right\|+2 \lambda_{1}^{-m+1} R_{0}^{2} M^{\prime}(v) \lambda_{j}^{\frac{m}{2}}\left(\left\|\nabla^{m} \xi_{1 j}\right\|\left\|\eta_{2 j}\right\|+\left\|\nabla^{m} \eta_{1 j}\right\|\left\|\eta_{2 j}\right\|\right) \\
\leq & \frac{1-\mu_{0}}{2} \lambda_{j}^{\frac{m}{2}}\left(\left\|\nabla^{m} \xi_{1 j}\right\|^{2}+\left\|\nabla^{m} \eta_{1 j}\right\|^{2}+\left\|\xi_{2 j}\right\|^{2}+\left\|\eta_{2 j}\right\|^{2}\right) \\
& +\lambda_{1}^{-m+1} R_{0}^{2} S_{0} \lambda_{j}^{\frac{m}{2}}\left(2\left\|\nabla^{m} \xi_{1 j}\right\|^{2}+2\left\|\xi_{2 j}\right\|^{2}+2\left\|\eta_{2 j}\right\|^{2}+2\left\|\nabla^{m} \eta_{1 j}\right\|^{2}\right) \\
= & \frac{1-\mu_{0}}{2} \lambda_{j}^{\frac{m}{2}}+2 \lambda_{1}^{-m+1} R_{0}^{2} S_{0} \lambda_{j}^{\frac{m}{2}},
\end{aligned}
$$

where $\mu_{0} \in[0,1]$. 
If $\left\|\xi_{2 j}\right\|^{2}+\left\|\eta_{2 j}\right\|^{2}=0$, then $P_{n_{1}} \leq-\frac{\varepsilon}{4} n_{1}, \quad q_{n_{1}}<0$.

By Lemma VI 6.3 of [8], Young's inequality and existing $\mu_{0}, s_{0}$ satisfying $\frac{1-\mu_{0}+4 S_{0} \lambda_{1}^{-m+1} R_{0}^{2}}{2} \lambda_{j}^{\frac{m}{2}}-\frac{\beta}{8} r \lambda_{j}^{m}-\frac{\varepsilon}{8} \leq 0$, we obtain

if $\left\|\xi_{2 j}\right\|^{2}+\left\|\eta_{2 j}\right\|^{2}=r \neq 0$, then

$$
\begin{aligned}
p_{n_{1}}(s)=\sum_{j=1}^{n_{1}}\left(-p(\varphi(s))+\Gamma_{1}(\varphi(s))+\Gamma_{2}(\varphi(s)) y_{j}(s), y_{j}(s)\right)_{E_{0}} \\
\leq \sum_{j=1}^{n_{1}}\left(-\frac{\varepsilon}{4}-\frac{\beta}{4}\left\|\nabla^{m} \xi_{2 j}\right\|^{2}-\frac{\beta}{4}\left\|\nabla^{m} \eta_{2 j}\right\|^{2}+\left(\frac{8 C_{45}^{2}}{\beta}\left\|A^{\frac{m}{2} \delta} \xi_{1 j}\right\|^{2}+\frac{\beta}{8}\left\|\nabla^{m} \xi_{2 j}\right\|^{2}\right.\right. \\
\left.\left.+\frac{8 C_{45}^{2}}{\beta}\left\|A^{\frac{m}{2}} \delta \eta_{1 j}\right\|^{2}+\frac{\beta}{8}\left\|\nabla^{m} \eta_{2 j}\right\|^{2}\right)+\frac{1-\mu_{0}}{2} \lambda_{j}^{\frac{m}{2}}+2 \lambda_{1}^{-m+1} R_{0}^{2} S_{0} \lambda_{j}^{\frac{m}{2}}\right) \\
=\sum_{j=1}^{n_{1}}\left(\frac{1-\mu_{0}}{2} \lambda_{j}^{\frac{m}{2}}+2 \lambda_{1}^{-m+1} R_{0}^{2} S_{0} \lambda_{j}^{\frac{m}{2}}-\frac{\beta}{8} \lambda_{j}^{\frac{m}{2}}\left(\left\|\xi_{2 j}\right\|^{2}+\left\|\eta_{2 j}\right\|^{2}\right)-\frac{\varepsilon}{4}\right)+\frac{32 C_{45}^{2}}{\beta} \sum_{j=1}^{n_{1}} \mu_{j}^{\delta-1} \\
=\sum_{j=1}^{n_{1}}\left(\frac{1-\mu_{0}}{2} \lambda_{j}^{\frac{m}{2}}+2 \lambda_{1}^{-m+1} R_{0}^{2} S_{0} \lambda_{j}^{\frac{m}{2}}-\frac{\beta}{8} \lambda_{j}^{\frac{m}{2}} r-\frac{\varepsilon}{4}\right)+\frac{32 C_{45}^{2}}{\beta} \sum_{j=1}^{n_{1}} \mu_{j}^{\delta-1} \\
\leq-\frac{\varepsilon}{8} n_{1}+\frac{\rho}{2} \sum_{j=1}^{n_{1}} \mu_{j}^{\delta-1},
\end{aligned}
$$

where $0<r \leq 1, \rho=\frac{64 C_{45}^{2}}{\beta}$.

If $\frac{\varepsilon}{4 \rho} \geq \frac{1}{n_{1}} \sum_{j=1}^{n_{1}} \lambda_{j}^{\delta-1}$ hold, then

$$
q_{n_{1}}=\liminf _{t \rightarrow \infty} \sup _{\tau \in R} \sup _{\Phi \subset E_{0}} \sup _{\varphi(\tau) \in \mathrm{A}} \frac{1}{t} \int_{\tau}^{\tau+t} p_{n_{1}}(s) \mathrm{d} s \leq-\rho n_{1}\left(\frac{\varepsilon}{4 \rho}-\frac{1}{n_{1}} \sum_{j=1}^{n_{1}} \lambda_{j}^{\delta-1}\right)<0 .
$$

Thus, by Lemma 4 of (S. Zhou, 1999 [9]), we obtain (3.39) and (3.40). The proof is completed.

\section{Conclusion}

In this paper, we study estimation of the upper bounds of Hausdorff dimension and Fractal dimension of the global attractor for a class of Higher-order coupled Kirchhoff-type equations. In the process of research, we have avoided further restriction by taking the overall treatment of $M(s)$ item, thus making the application of this model more extensive. Although the theoretical derivation of the beam vibration model is not combined with the application in real life, it is necessary to combine with practical application to further study.

\section{Acknowledgements}

We express our heartful thanks to the anonymous reader for his/her careful reading of this paper. We hope that we can obtain valuable comments and advices. These 
contributions vastly improved the paper and making the paper better.

\section{References}

[1] Lin, G.G. and Yang, S.M. (2017) The Global Attractors for the Higher-Order Coupled Kirchhoff-Type Equations with Strongly Damped Terms and Source Terms. European Journal of Mathematics and Computer Science, 4.

[2] Sun, Y.T., Gao, Y.L. and Lin, G.G. (2016) The Global Attractors for the Higher-Order Kirchhoff-Type Equation with Nonlinear Strongly Damped Term. International Journal of Modern Nonlinear Theory and Application, 5, 203-217. https://doi.org/10.4236/ijmnta.2016.54019

[3] Chen, L., Wang, W. and Lin, G.G. (2016) The Global Attractors and Their Hausdorff and Fractal Dimensions Estimation for the Higher-Order Nonlinear Kirchhoff-Type Equation. International Journal of Modern Communication Technologies and Research, 4, 6-12.

[4] Lin, G.G. and Gao, Y.L. (2017) The Global and Exponential Attractors for the Higher-Order Kirchhoff-Type Equation with Strong Linear Damping. Journal of Mathematics Research, 9, 145-167. https://doi.org/10.5539/jmr.v9n4p145

[5] Lin, G.G. (2011) Nonlinear Evolution Equation. Yunnan University Press, Kunming.

[6] Ye, Y.J. (2013) Global Existence and Energy Decay Estimate of Solutions for a Higher-Order Kirchhoff Type Equation with Damping and Source Term. Nonlinear Analysis. Real World Application, 14, 2059-2067. https://doi.org/10.1016/j.nonrwa.2013.03.001

[7] Teman, R. (1989) Infinite Dimensional Dynamics Systems in Mechanics and Physics. Springer, New York.

[8] Wu, J.Z. and Lin, G.G. (2009) The Global Attractor of the Bossinesq Equation with Damping Term and Its Dimension Estimation. Journal of Yunnan University, 31, 335-340.

[9] Zhou, S. (1999) Global Attractor for Strongly Damped Nonlinear Wave Equations. Functional Differential Equations, 6, 451-470. https://doi.org/10.1006/jmaa.1999.6269 